\title{
Individuelles Verhalten und sozialpolitische Anreize: Das fordernde Element im Wohlfahrtsstaat
}

\begin{abstract}
Viele sozialpolitische Maßnahmen des letzten Jahrzehnts sind weder als Verteilungspolitik noch als Daseinsvorsorge treffend charakterisiert. Stattdessen beziehen sie sich - fördernd und fordernd - auf das Verhalten und die Entscheidungen der einzelnen Bürger und versuchen, auf diese einzuwirken. Sind diese Anreiz-Elemente in der Sozialpolitik wirklich neu? Und was macht ihren besonderen Charakter in theoretischer Hinsicht aus? Die Antwort auf diese Fragen hat Folgen für unser Verständnis der jüngsten Sozialreformen und sie hilft, auch sozialpolitischen Wandel generell besser zu verstehen.
\end{abstract}

\section{Einleitung}

Die Formel vom „Fördern und Fordern“ sollte zunächst nur ein griffiges Konzept für einen Politikwechsel in der Arbeitsmarktpolitik vorstellen. In welchem Ausmaß das Motto zur Chiffre für eine veränderte Akzentsetzung in der Sozialpolitik der Bundesrepublik Deutschland insgesamt werden würde, konnte für die Macher der Agenda 2010 kaum absehbar gewesen sein. Fordernde Elemente, d.h. relativ deutliche Verhaltenserwartungen an die Bürger, blieben nicht auf die Arbeitsmarktpolitik beschränkt, sondern traten auch in einer ganzen Reihe anderer sozialpolitischer Politikfelder stärker in den Vordergrund. Nirgends ist das deutlicher sichtbar als in der Familienpolitik: Bestimmte Lebensweisen - Familiengründung, Kinderbetreuung in Kindertagesstätten, Erwerbstätigkeit beider Eltern - werden hier sehr dezidiert und generös sozialpolitisch alimentiert, doch andere eben nicht. Im Zuge dessen steht neuerdings auch ganz offen die Frage im Raum: „Wem gehört [sic!] die Familie der Zukunft?" (Bertram et al. 2006), und Regierungsbeamte sprechen freimütig vom "ökonomischen Charme der Familie“ (Ristau 2005). Sozialpolitisches „Fördern und Fordern" formuliert sehr klar und explizit ein instrumentelles Interesse an privaten Lebensentwürfen und individuellen Verhaltensweisen. Das befremdet etwas, und es stellt uns sowohl in der politischen Beurteilung als auch im theoretischen Verständnis vor neue Herausforderungen.

Das Anliegen dieses Beitrags ist es daher, den besonderen Charakter des Förderns und Forderns in der Sozialpolitik historisch vergleichend darzustellen und theoretisch zu reflektieren. Die Leitfrage der folgenden Überlegungen lautet: Wie kann man Anreizelemente in der Sozialpolitik konzeptionell fassen, und was folgt daraus für die Erklärung wohlfahrtsstaatlicher Entwicklung? Nach einer kurzen Illustrierung und Einordnung des Prinzips von Leistung und Gegenleistung im Wohlfahrtsstaat (Abschnitt 2) werden zunächst die expliziter fordernden Elemente der auf Nachhaltigkeit bedachten Sozialpolitik der letzten Jahre dargestellt (Abschnitt 3.1). Der heutigen Kürzungspolitik wird in Abschnitt 3.2 die Sozialpolitik während der "goldenen Jahre“ wohlfahrtsstaatlichen Wachstums gegenübergestellt, die vor allem soziale Rechte erweiterte. Der Vergleich zeigt, dass sich die beiden Perioden nicht so sehr durch die Existenz sozialpolitischer Anreizelemente unterscheiden, sondern lediglich durch die Erscheinungsformen und Ziele der sozialpolitischen Forderungen. An die historisch vergleichende Darstellung schließen sich einige Überlegungen zur theoretischen Konzeptionalisierung an (Abschnitt 4): Es werden die Schwierigkeiten dargelegt, die der theoretischen Konzeptionalisierung sozialpolitischer Anreizpolitik im Rahmen der vorherrschenden Paradigmen der Wohlfahrtsstaatsforschung entgegenstehen, und es werden einige Vorschläge gemacht, wie man sich produktiv mit dem sozialpolitischen Fordern theoretisch auseinandersetzen kann. Schließlich wird das Potenzial dieser alternativen Sichtweise auf dem Weg zu einer Erklärung wohlfahrtsstaatlichen Wandels aufgezeigt.

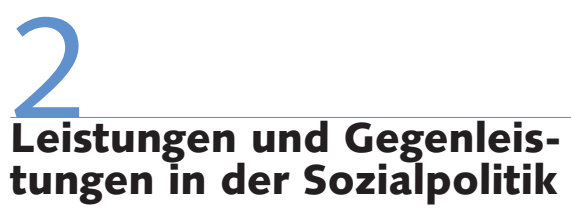

Grundsätzlich neu ist die Vorstellung von der Sozialpolitik als einer Struktur von Leistungen, Gegenleistungen und Verhaltensanforderungen nicht. Im Gegenteil: Die den deutschen Wohlfahrtsstaat prägende Sozialversicherung betont das Beitragsprinzip und mit ihm die enge Verbindung zwischen Sozialleistungen und Vorleistungen. Fürsorgesysteme sind weithin bekannt für ihren sozialarbeiterischen und sozial-disziplinierenden Charakter (Sachße/ Tennstedt 1986), und sie sind durchzogen von Versuchen, mithilfe von staatlichen

\footnotetext{
Margitta Mätzke, Ph.D., ist wissenschaftliche Mitarbeiterin an der Universität Göttingen. Arbeitsschwerpunkte: Vergleichende Wohlfahrtsstaatsforschung, Sozialpolitische Gesetzgebung, Interessenvermittlung in der Arbeits- und Sozialpolitik. e-mail:mmaetzk@gwdg.de
} 
Sozialleistungen das Verhalten der unteren Bevölkerungsschichten zu regulieren (Piven/Cloward 1971). Versorgungssysteme sind zum großen Teil auf eine besondere Beziehung zwischen Leistungsbeziehern und dem Staat aufgebaut und teilweise als Gratifikation für besondere Leistungen konzipiert (Skocpol 1992). Obwohl also der Aspekt der Gegenleistung theoretisch wie praktisch in der Sozialpolitik immer präsent ist, gerät er in der politischen und theoretischen Wahrnehmung zuweilen aus dem Blickfeld. Lange stand er im Schatten eines Bildes vom Wohlfahrtsstaat, in dem es vor allem um Daseinsvorsorge, Dekommodifizierung (Esping-Andersen 1990), Befreiung aus Abhängigkeiten und Verteilungspolitik ging, der Schwerpunkt des Interesses also eindeutig auf der Leistungsseite lag. Die Rede vom Fördern und Fordern in der Sozialpolitik ruft den Aspekt der Gegenleistung für empfangene Unterstützung in Erinnerung.

Darüber hinaus betont sie eine sehr viel direktere, persönlichere Ansprache, mit der Sozialpolitiker heute ihren Bürgern gegenübertreten. Sie sprechen, wie Adalbert Evers (2008, S. 230) es formuliert, „eine starke Sprache" und formulieren Verhaltenserwartungen und Präferenzen über erwünschte Lebensentwürfe: Eigenverantwortliches Vorsorgen fürs Alter wird sozialpolitisch gefördert, lebenslanges Lernen, berufliche Mobilität und „aktives Altern“ avancieren zu Schlagworten in der öffentlichen Rhetorik (Evers 2008, S. 241). Es sind nicht die abstrakten Umverteilungsströme, Sozialversicherungsbeiträge oder Anwartschaften, mit denen der Wohlfahrtsstaat hier in Erscheinung tritt, sondern hier sind die Bürger mit ihren individuellen täglichen Verhaltensweisen und Entscheidungen gefordert. Oft bleibt es bei der Rhetorik. In den Bereichen, in denen sich das Interesse an Gegenleistungen jedoch in sozialpolitischen Reformen niederschlägt, beobachten wir Politikwechsel mit teilweise einschneidenden Maßnahmen. In der Arbeitsmarktpolitik ist das augenfällig. Erfolg und vor allem Verteilungseffekte der Hartz-Reformen mögen umstritten sein, doch die aktivierenden Elemente und die Aufforderung zur aktiven Mitarbeit der Arbeitsuchenden haben hier deutlich an Bedeutung gewonnen (Kemmerling/Bruttel 2006, S. 97f.). In der Rentenpolitik wird die private Altersvorsorge zurzeit sozialpolitisch gefördert, bleibt aber freiwillig (Hinrichs 2004). In der Familienpolitik spielt neuerdings deutlicher als man es in der Bundesrepublik gewöhnt ist, das Motiv der Geburtenförderung eine Rolle. Darüber hinaus wird die Familie ganz offen in den Dienst ökonomischer Nebenziele gestellt (Ostner 2008): Erwerbstätigkeit beider Eltern wird sozialpolitisch gefördert, und es sind vor allem Akademikerpaare, die bei den neuen familienpolitischen Aktivitäten im Zentrum des Interesses stehen (Henninger et al. 2008). Das Aktivierungsparadigma enthält mit seiner Betonung von Eigenverantwortung und Gegenleistungen also relativ klare Erwartungen an das Verhalten der Bürger.

Viel stärker als wir es aus der Fürsorgepolitik gewöhnt sind, beziehen sich die erzieherischen Absichten der Sozialpolitik nicht vorrangig auf Randgruppen, sondern sie zielen durchaus in die Mitte der Gesellschaft; auch hier werden bestimmte Verhaltensweisen sozialpolitisch gefördert und andere ignoriert. Im Zentrum der sozialpolitischen Forderungen stehen nicht Interessengruppen oder Organisationen, sondern das Verhalten und die Entscheidungen der einzelnen Bürger. Diese können sich entscheiden, Kinder zu haben, sie können auf ihre Gesundheit achten, in ihr berufliches Fortkommen Zeit und Mühe investieren; sie können lebenslange, stetige berufliche Karrieren mit guten (steuerpflichtigen) Einkommen verfolgen und in ihrem Beruf Einsatz zeigen, dadurch vielleicht sogar Arbeitsplätze schaffen; sie können Berufssoldaten werden, ihre Angehörigen pflegen und ihren Kindern eine gute Ausbildung ermöglichen. Sie könnten all das aber auch bleiben lassen. Und die große Nervosität, die unter politischen Eliten entsteht, wenn relevante Minderheiten sich entscheiden, keine Kinder zu haben, keine ordentliche berufliche Zukunft ins Auge zu fassen, sich nicht um das Wohl ihrer Angehörigen zu sorgen, den politischen Institutionen des Landes distanziert oder ablehnend gegenüberzustehen, zeugt von der Wirkungsmacht der individuellen Verhaltensweisen.

Nicht nur in der politischen Beurteilung, sondern auch in der theoretischen Deutung stellt uns das fordernde Element in der Sozialpolitik vor neue Herausforderungen. In der Deutung neuer Tendenzen in der Sozialpolitik ist viel intellektuelle Energie in Theorien der Erosion staatlicher Macht, des Rückzugs des Staates und der Privatisierung ehemals öffentlicher Verantwortlichkeiten geflossen. In vielen
Bereichen der fordernden Sozialpolitik lässt sich jedoch kein Rückzug, sondern im Gegenteil ein zunehmendes Maß an staatlicher Intervention beobachten (Baldwin 2005). Um zu bestimmen, ob wir es tatsächlich mit einer wachsenden Präsenz des Staates im Leben der Bürger zu tun haben, wird im Folgenden die heutige, auf Nachhaltigkeit bedachte Sozialpolitik mit der Expansionsphase der Sozialpolitik nach dem Zweiten Weltkrieg kontrastiert. Das hilft zu klären, ob sich fordernde Elemente einzig als Reflex auf Überlastungstendenzen verstehen lassen, im Zuge derer wachsende ehemals staatliche Aufgabenbereiche in private Verantwortlichkeit übertragen werden sollen.

Dass diese Argumentationsfigur den Kern des jüngeren sozialpolitischen Richtungswechsels nicht trifft, zeigen die folgenden Ausführungen. Vielmehr war es auch in der expansiven Nachkriegssozialpolitik ein zentrales Anliegen, das Verhalten der Bürger durch sozialpolitische Maßnahmen zu lenken und ein stückweit zu normieren, ebenso wie wirtschaftspolitische Nebenziele das sozialreformerische Geschehen stark geprägt haben. Die Nachkriegsjahrzehnte hatten eine prägende Kraft für die Sozialpolitikentwicklung (Evers 2008, S. 242). Vor ihrem Hintergrund treten Akzentverschiebungen der jüngeren Sozialreformen deutlich hervor, doch das sollte die konstanten Motive sozialstaatlicher Entwicklung nicht zu sehr in den Schatten stellen. Zu diesen Konstanten sozialreformerischer Aktivität zählt die verhaltenslenkende, fordernde Absicht, die in den Entscheidungen der Sozialreformer damals wie heute Pate gestanden hat.

\section{Sozialpolitische Anreize im Vergleich}

\subsection{FÖRDERN UND FORDERN ALS GEBOT DER NACHHALTIGEN SOZIALPOLITIK}

Die jüngsten Reformen der Sozialpolitik in der Bundesrepublik Deutschland stehen im Zeichen leerer öffentlicher Kassen und anhaltender Arbeitsmarktkrisen. Ein zunehmendes Maß an privat zu übernehmender Risikovorsorge soll das Aufgabenspektrum der staatlichen Sozialpolitik bewältigen helfen, einen Ausweg aus den 
finanziellen Problemen zeigen und den Bürgern als legitim und gerecht vermittelt werden. Die ersten Schritte in Richtung Privatisierung von Lebensrisiken schienen nicht so einschneidend. Sie betrafen die traditionell relativ generös ausgestatteten Kernbereiche der Sozialversicherung, das Gesundheitswesen und die Alterssicherung; und es waren kleine Schritte: Leistungsausgrenzungen in Randbereichen der Gesundheitsversorgung und moderate Zuzahlungen sowie die Förderung der privaten Altersvorsorge. In ihrer Summe stellen diese graduell wachsenden Elemente privat zu erbringender Vorsorge eine Verschiebung von Risiken in den Bereich der privaten Vorsorge dar, die sich vor allem auf lange Sicht auswirkt. In den Kategorien dieses Beitrages liegt in den sozialpolitischen Anreizen - der Riesterförderung in der Alterssicherung, den Zuzahlungen oder der Praxisgebühr in der Gesundheitspolitik - das fordernde Element in der Sozialpolitik: In ihnen liegt eine Aufforderung staatlicher Sozialreformer an ihre Bürger, aktiv bei der Vermeidung und Finanzierung sozialer Risiken mitzuwirken.

Die renten- und gesundheitspolitischen Entwicklungen sind jedoch vor allem auch deshalb wichtig, weil sie die Vorstellung der Risiko-Privatisierung graduell etablierten. Private Altersvorsorge wird seit der Riester-Reform im Jahr 2001 sozialpolitisch gefördert, doch sie gilt seitdem als Teil des Alterseinkommens; der „verdiente Ruhestand“ wird damit nicht mehr allein öffentlich gewährleistet (Hinrichs 2004). Arbeitslosigkeit und Armut waren die nächsten Risikotatbestände, für die ein größeres Maß an privat zu tragender Verantwortung eingefordert wurde. Das zu etablieren, ist die einschneidende Bedeutung der Hartz-Reformen der Jahre 2003 und 2004. Erstmals als „Fördern und Fordern“ explizit so benannt, verstärkten die Hartz-Reformen die (mehr oder weniger erfolgreichen) aktivierenden Elemente in der Arbeitsmarktpolitik drastisch. In ihnen wurde die stärkere Mitwirkung beim Qualifikationserwerb und der Vermittlung in den ersten Arbeitsmarkt als Pflicht der Leistungsempfänger stark betont und durch Sanktionsdrohungen untermauert. Konzipiert als Kehrseite des Aktivierungsversprechens - oft aber de facto eine einseitige Sparmaßnahme - ist das Risiko der Langzeitarbeitslosigkeit seit 2004 in der deutschen Arbeitslosenversicherung nicht mehr versichert.
Workfare-Konzepte, die im Zuge der Hartz-Reformen in Deutschland an Bedeutung gewonnen haben, enthalten eine starke Präsenz staatlich gesetzter Normen im Leben der Leistungsempfänger. Beschäftigungs- und Arbeitsmarktpolitik operierten allerdings schon seit ihren Anfängen mit ziemlich klaren Vorstellungen über erwünschtes, zu förderndes Erwerbsverhalten der Bürger. Arbeitsmarktprobleme sollten immer wieder von der Angebotsseite her, d.h. durch Einflussnahme auf das Erwerbsverhalten der Bürger gelöst werden. Zur Bewältigung der Massenarbeitslosigkeit sollten lange Zeit möglichst viele Personen vom Arbeitsmarkt ferngehalten werden. Frühverrentungsregelungen, sehr lange höhere Ausbildungsgänge und nicht zuletzt eine am Leitbild der nicht-erwerbstätigen Mutter festhaltende Familienpolitik - all diese Maßnahmen sollten in der Vergangenheit bestimmten Personengruppen (mehr oder weniger dauerhafte) Alternativen zur Erwerbstätigkeit anbieten.

Dieses Reaktionsmuster der Stilllegung von Arbeitsangebot war lange Zeit die politische Antwort auf das Problem der Massenarbeitslosigkeit (Streeck 2001), doch es stieß nach der Jahrtausendwende endgültig an die Grenzen seiner Finanzierbarkeit. Nicht Arbeitslosigkeit per se, sondern Inaktivität-Nicht-Erwerbstätigkeit - wurde zum hauptsächlich zu bekämpfenden Übel und eine hohe Beschäftigungsquote immer mehr Hauptziel und Hauptbezugspunkt einer aktivierenden Beschäftigungspolitik. Das arbeitsmarktpolitische Anliegen, einmal erworbene Qualifikationen durch Vermittlung in ausbildungsadäquate Berufe zu erhalten - eines der grundlegenden Ziele des alten Arbeitsförderungsrechts ${ }^{1}$ - ist dem Bestreben gewichen, gerade die Langzeitarbeitslosen praktisch um jeden Preis in Beschäftigung auf dem ersten Arbeitsmarkt zu vermitteln.

Das starke familienpolitische Engagement der Bundesregierung in jüngerer Zeit muss man als Teil dieses breiteren, im Kern arbeitsmarktpolitischen Strategiewechsels auffassen. Zeitgleich mit der Neuausrichtung der Arbeitsmarktpolitik am Ziel einer hohen Beschäftigungsquote begann eine familienpolitische Diskussion, in der Besorgnis über niedrige Geburtenzahlen immer mehr ins Zentrum rückte und die immer öfter die Befunde der international vergleichenden Forschung aufgriff, nach denen es der Geburtenrate abträg- lich ist, wenn man Mütter aus dem Beruf herausdrängt und damit Frauen in eine Entscheidung zwischen Kindern und Beruf hineinzwingt (Schoppa 2005). Seit der zweiten Legislaturperiode der Rot-Grünen Bundesregierung wird der demografische Wandel in einer Weise thematisiert, die das Thema Geburtenförderung offen auf die Agenda setzt (Rürup/Gruescu 2003), was letztlich auch in die weit reichenden familienpolitischen Reformen eingemündet ist. In dieser Reformkonzeption kristallisiert sich die bessere Vereinbarkeit von Familie und Beruf, vor allem für Mütter, als zentrales Element heraus. Das lässt sich zum Teil als geburtenfördernde Politik verstehen, die es Frauen ermöglichen soll, ihre Kinderwünsche zu verwirklichen, ohne dadurch alle beruflichen Ambitionen auf Eis legen zu müssen.

Im Kontext der arbeitsmarkt- und sozialpolitischen Gesamtstrategie wird aber in der neuen sozialpolitischen Zielvorstellung der Zweiverdienerhaushalte noch eine andere Logik deutlich: In dem $\mathrm{Maße}$, in dem Beschäftigungsverhältnisse immer unsicherer und Armutsrisiken staatlich immer lückenhafter abgesichert werden, sind zwei Erwerbseinkommen im Haushalt die potenteste private Vorsorge gegen diese Risiken. Armut in der Familie wird somit auch immer einhelliger aufgefasst als „eine Folge ihres nicht ausgeschöpften Erwerbspotenzials und [sei] daher zunächst durch die Aktivierung der nichterwerbstätigen Mutter zu bekämpfen“ (Ostner 2006, S. 195). Private Unterstützung in Familien und Lebensgemeinschaften wird somit immer wichtiger als Ressource in den wachsenden Lücken des sozialen Netzes. Zunehmend wird sie explizit einkalkuliert und sozialpolitisch gefördert. Damit aber ermöglicht (Vollzeit-)Erwerbstätigkeit diese Art privater Unterstützung, und wird deshalb immer ausgeprägter zur sozialpolitischen Norm für alle Erwachsenen. Bei Abweichungen von dieser Norm, die als legitim erachtet werden - wenn etwa statt der Erwerbsarbeit wichtige Aufgaben in der Familie erfüllt werden ${ }^{2}-$, werden die Sozialleistungen als Kompensation für entgangenes

1 Im Arbeitsförderungsgesetz (AFG) von 1969 wird in der Präambel noch das Ziel, Arbeitslosigkeit und unterwertige Beschäftigung zu vermeiden, formuliert.

2 Pflege-, Betreuungs- und Erziehungstätigkeiten, frühkindliche Förderung des Nachwuchses. 
Erwerbseinkommen ausgestaltet. Das bekräftigt den Status der (Vollzeit-)Erwerbstätigkeit als sozialpolitische Norm, und es unterstreicht, dass Erwerbsunterbrechungen nur temporär (und kurzzeitig) für legitim erachtet werden. Insgesamt also verliert die Familie zwar einerseits ihre Rolle als Bezugspunkt vieler abgeleiteter sozialpolitischer Leistungsansprüche ${ }^{3}$, sie wird aber andererseits gleichzeitig als „Rückversicherung“ immer wichtiger. Diesen Prozess hat Ilona Ostner (2006, S. 189f.) als simultane „Entfamilisierung“ und „Re-Familisierung“ charakterisiert.

Aus der Kombination von Zielen, die nicht so einfach in Einklang zu bringen sind - hohe Erwerbsbeteiligung, auch unter Eltern sehr kleiner Kinder, doch gleichzeitig auch in Familien zu erbringende Pflege-, Erziehungs- und Unterstützungsleistungen-, erklärt sich sowohl die zentrale Rolle der Familienpolitik als auch die Engführung der Familienpolitik auf Vereinbarkeitspolitiken in der Gesamtkonstellation sozialpolitischer Reformbestrebungen im letzten halben Jahrzehnt. Maßnahmen wie das Elterngeld oder der Ausbau der Betreuungsplätze für Kleinkinder sind stark expansiv, während in praktisch allen anderen Bereichen der Sozialpolitik versucht wurde (wenn auch nicht immer erfolgreich), Kosten einzusparen. Wie auch in der Arbeitsmarktpolitik veränderte sich in der Familienpolitik die Leitvorstellung sozialpolitischer Intervention. Familienpolitik und Arbeitsmarktpolitik richteten also ihre Maßnahmen auf inhaltlich neue Ziele - neue sozialpolitisch kommunizierte Verhaltensnormen.

\subsection{AUSBAU DES SOZIALSTAATS IM DIENST WIRTSCHAFTLICHER UND POLITISCHER ZIELE}

Sind Anforderungen an individuelles Verhalten, wie wir sie in der Sozialpolitik neuerdings so deutlich beobachten können, immer Auswüchse der Kürzungspolitik, d.h. Versuche, sich in Zeiten knapper Kassen öffentlicher Aufgaben zu entledigen? Der vergleichende Blick auf die Expansionsphase der bundesdeutschen Sozialpolitik zeigt, dass sozialpolitische Maßnahmen auch unter permissiven fiskalischen Bedingungen Anreizelemente enthalten und von Normen über erwünschte Lebensweisen mitgeprägt sind. Das stellt die Verteilungsdimensionen der Sozialpolitik nicht in
Abrede, ebenso wenig wie oben behauptet werden sollte, dass die heutige Sozialpolitik frei von verteilungspolitischen Dimensionen ist. Die heutigen grundlegenden Reformen sind zudem das Ergebnis einer Entwicklung, in der sich das Bild von Erwerbsarbeit, Familie und auch die Modelle und Realitäten erfolgreicher Wirtschaftspolitik graduell verändert haben. Auch das soll nicht geleugnet werden. Der Vergleich mit der Nachkriegssozialpolitik ist jedoch gerade deshalb aufschlussreich, weil er die maximal unterschiedlichen wirtschaftlichen und fiskalischen Bedingungen und die krassen Unterschiede in den Kontextbedingungen der Sozialpolitik beleuchtet. So kann man sehen, wie grundverschiedene Bedingungen auf das Ausmaß und die inhaltlichen Schwerpunkte sozialpolitischer Anreizpolitik wirken.

Unmittelbar nach dem Krieg diente sozialpolitische Expansion unter anderem dazu, die Bundesrepublik in ihrer „Gründungskrise" (Hockerts 1986) innenpolitisch zu stabilisieren und sozial zu befrieden. Die teilweise sozialpolitisch erkaufte Legitimität der jungen Demokratie sollte das politische System in den Augen seiner Staatsbürger attraktiv machen - attraktiver auch als das alternative Wirtschafts- und Gesellschaftsmodell in Ostdeutschland. Das Ausmaß und der Zuschnitt des rasch wachsenden Sozialstaates waren somit von der Überlegung geprägt, „dass die Sozialreform die außenpolitische Grundlegung des neuen Staates durch eine innenpolitische Fundamentierung ergänzen sollte“ (Hockerts 1980, S. 244). De facto hat es im Rahmen der Kriegsfolgengesetzgebung in der unmittelbaren Nachkriegszeit Einkommenstransfers in großem Ausmaß gegeben, die Massennotstände verhindert haben und die Situation innenpolitisch befrieden konnten. Diese Leistungen entwickelten sich allerdings wenig systematisch und auch wenig kontrolliert, eine Situation, die in den 1950er Jahren zunehmend Unzufriedenheit hervorrief und den Anstoß zum Vorhaben einer großen Sozialreform gab. Gemeint war damit zwar vorwiegend nur die Reform der sozialen Leistungen, bald eng geführt auf eine Reform der gesetzlichen Rentenversicherung (Hockerts 1980), doch auch die war nicht frei von Elementen der fordernden Sozialpolitik.

Vorrangig war in der Nachkriegszeit somit die politische Integrationsaufgabe. Hierbei waren Einkommenstransfers an Nicht-Erwerbstätige und vor allem die
Rentenpolitik zentrale Betätigungsfelder. Die Erkenntnis, „dass der moderne Staat seinen Bürgern das Gefühl der Sicherheit für das Alter geben muss - das Gefühl, nach einem Leben voller Arbeit nicht, deklassiert' zu werden“" (Jantz 1977, S. 110f.) - war für viele Arbeiter und Angestellte vor dem Hintergrund katastrophal geringer Altersrenten von großer Bedeutung. Strukturprinzipien der 1957er Rentenreform wie Beitragsbezogenheit der Rente, Beibehaltung der berufsständischen Gliederung der Versicherungsträger und dynamisierte, d.h. vor Erosion im Verlauf der wirtschaftlichen Entwicklung geschützte Leistungen entsprachen einem in den 1950er Jahren verbreiteten Bedürfnis nach sozialer Sicherheit (Braun 1978), doch sie waren in der Art, in der sie dies taten, nicht neutral. Es wurde nicht allen Bürgern gleichermaßen eine großzügige Rente gewährt, sondern es wurden höchst differenzierte, auf individueller Leistung aufbauende Ansprüche geschaffen. Sie knüpften an bestimmte Verhaltensweisen wie qualifizierte Berufstätigkeit in langfristigen Beschäftigungsverhältnissen an.

Galt das zunächst für die Rentenversicherung, so wurde es strukturprägend für viele Sozialleistungen (Jantz 1977). Im Verlauf der 1960er Jahre unter dem Eindruck erster konjunkturell und strukturell bedingter Arbeitsmarktschwierigkeiten nahm die Bedeutung beruflicher Qualifikation noch zu. Das Arbeitsförderungsgesetz (AFG) von 1969 schuf ein breites Spektrum von Maßnahmen der aktiven Arbeitsmarktpolitik, die Arbeitnehmern Angebote zur beruflichen Weiterbildung machten. Der aktiven Arbeitsmarktpolitik nach dem AFG fehlte jedoch der Verpflichtungscharakter, der später die aktivierende Beschäftigungspolitik prägen würde. Indem die Sozialversicherung auf individuellen beruflichen Erfolg als Grundlage vieler Sozialleistungen zugeschnitten war und damit nur bestimmte Lebensweisen sozialpolitisch honorierte, unterstützte sie nicht nur den Gesellschaftsentwurf der frühen Bundesrepublik, sondern sie bildete auch in ökonomischer Hinsicht eine Stütze der sich entwickelnden Wirtschaftsordnung

\footnotetext{
3 Abgeleitete Sozialversicherungsansprüche, Unterhaltsansprüche nach Ehescheidungen, wenn keine Kinder im Spiel sind, Diskussionen um den Wegfall der beitragsfreien Krankenversicherung von nicht erwerbstätigen Ehepartnern (vgl. Ostner 2006, S. 189).
} 
(vgl. Estévez-Abe et al. 2001; Manow 2001). Ulrich Mückenberger (1985) hat den Begriff des Normalarbeitsverhältnisses in die sozialpolitische Literatur eingeführt und damit die sowohl Normalität widerspiegelnde als auch norm-setzende Rolle der sozialpolitischen Regulierung von Arbeitsverhältnissen betont.

Die Normalitätsannahmen in Bezug auf individuelles Erwerbsverhalten und (männliche) Berufsbiografien finden ihre Entsprechung in ähnlich stark ausgeprägten Leitvorstellungen über die Familie. In den 1950er Jahren wurden mit dem Kindergeld (1954) direkte Transferleistungen an kinderreiche Familien (wieder) eingeführt. Dieser „schichtspezifische Familienlastenausgleich", wie der konservative Familienminister Franz Josef Wuermeling ihn nannte, sollte ausdrücklich nicht zwischen Arm und Reich umverteilen (Moeller 1993, S. 121), ${ }^{4}$ sondern vorrangig zwischen Kinderlosen und kinderreichen Familien (Wuermeling 1956, S. 262). Ferner war er von der Vorstellung traditioneller Geschlechterrollen geprägt, und es ging vor allem um die Frage, wie man den Frauen ein Dasein als Hausfrau und Mutter ermöglichen könne (Schulz 1998, S. 130). Wenngleich man die Familie in der Nachkriegszeit als „unentbehrlichen Ordnungsfaktor" und „wichtigsten Erziehungsträger" der Gesellschaft (Wuermeling 1963, S. 85f.) ansah, wurde aktive Geburtenförderung in der sozialpolitischen Umsetzung (wenngleich nicht immer in der politischen Rhetorik) entschieden abgelehnt (Schulz 1998, S. 122).

Unzweifelhaft verbanden sich mit den sozialpolitischen Maßnahmen der Nachkriegszeit sowohl im Hinblick auf (männliches) Erwerbsverhalten als auch im Hinblick auf private Lebensentwürfe und die Rolle der Familie sehr dezidierte Erwartungen an ein bestimmtes Verhalten. Diese Erwartungen bezogen sich jedoch weniger auf quantitatives Bevölkerungswachstum oder Rekrutierung aller Arbeitsfähigen in die Erwerbstätigkeit, als vielmehr auf qualitative Aspekte der familiären, beruflichen und politischen Sozialisation. Zuverlässige Arbeitskräfte, Steuerzahler und Hausfrauen waren wesentliche Ressourcen im westdeutschen Wirtschaftswunder, ein wesentlicher Faktor auch in der erfolgreichen Konsolidierung der bundesdeutschen Demokratie, und auf sie hin waren viele sozialpolitische Leistungen zugeschnitten.

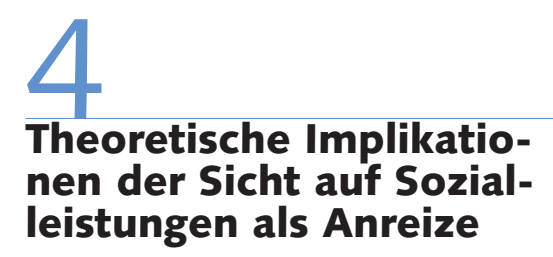

\subsection{KONSTANTEN DER FORDERNDEN SOZIALPOLITIK}

Fordernde Sozialpolitik ist somit nicht neu. Sie erscheint uns derzeit neu, und zuweilen befremdlich, weil sich die inhaltliche Ausrichtung der sozialpolitischen Anreizpolitik innerhalb der kurzen Zeitspanne eines Jahrzehnts relativ stark verändert hat. Die gesetzliche Rente verwandelte sich aus einer Lebensstandard sichernden in eine Grundsicherungsleistung. In der Arbeitsmarktpolitik verliert das Leitbild der lebenslangen qualifizierten Berufsarbeit an Geltung, und eine wachsende Zahl von Arbeitnehmern ist einer Beschäftigungspolitik ausgesetzt, die für sie den flexiblen Einsatz in nicht auf Dauer angelegten Jobs als langfristiges Schicksal ins Auge fasst. In der Familienpolitik vollzieht sich der Wandel vom Ernährermodell zur sozialpolitischen Förderung von (qualifizierter) Erwerbstätigkeit beider Eltern wahrscheinlich am schnellsten und am auffälligsten.

Trotz der zunehmenden Unsicherheit und trotz der Zunahme marktförmiger Elemente sind diese Entwicklungen mit dem Bild vom Rückzug des Staates aus umverteilenden Verbindlichkeiten (Höpner 2006, S. 17) und Liberalisierung (als Erosion nicht-marktförmiger Koordination) nicht sehr gut beschrieben. Die Vorstellungen vom Autonomieverlust der Sozialpolitiker (Evers 2008, S. 232f.; Trampusch 2006) oder von der Ökonomisierung der Sozialpolitik (Evers/Heinze 2008) liefern ebenfalls keine treffende Beschreibung der Veränderungen; der vergleichende Blick auf die frühere Phase der sozialpolitischen Entwicklung hat das gezeigt. Handlungsspielräume und Leitbilder der Sozialpolitik wurden zu allen Zeiten, selbst in den Jahren großer finanzieller Spielräume, von der wirtschaftspolitischen Umgebung stark geprägt. Intensive Debatten um makroökonomische Konsequenzen von Rentendynamisierung und Umlageverfahren, um das Kindergeld und den „Leistungslohn“ des männlichen Alleinverdieners, um das duale System der beruflichen Bildung und den Rechtsanspruch aufberufliche Weiter- bildung und um das Lohnabstandsgebot bei arbeitsmarktpolitischen Maßnahmen deuten an, dass von Autonomie der Sozialpolitik und von sozialpolitischer Gestaltung unabhängig von ihren ökonomischen Voraussetzungen und Konsequenzen auch unter den günstigsten finanziellen Bedingungen nicht die Rede sein konnte. ${ }^{5}$ Was veränderlich ist, ist die Art der wirtschaftspolitischen Nebenziele, nicht ihre Existenz.

Es werden auch die Autonomiespielräume und Wahlmöglichkeiten der Individuen nicht größer und vielfältiger. Jedoch sehen wir das Verhalten und die Lebensentwürfe der Leute von einem dichteren Netz öffentlicher Erwartungen und Verhaltensanforderungen umsponnen. Wirtschaftliche Nebenziele hat die Sozialpolitik also immer, und interventionistisch ist sie auch immer. Was sich wandelt, ist das unterlegte Modell der erfolgreichen Wirtschaft und der guten Gesellschaft. Dessen Einfluss auf sozialpolitische Anreizpolitik hat zu unterschiedlichen Zeiten unterschiedliche Erscheinungsformen, sein normsetzender Charakter tritt auch unterschiedlich deutlich zutage, ${ }^{6}$ und schließlich manifestiert er sich in verschiedenen Politikfeldern unterschiedlich. Dennoch ist das fordernde Element in der Sozialpolitik eine allgegenwärtige Tiefenstruktur der Leistungsgestaltung und Unterschiede an der Oberfläche der Erscheinungsformen sollten darüber nicht hinwegtäuschen.

\subsection{DER DOPPELCHARAKTER SOZIALER RECHTE}

Die vergleichende Wohlfahrtsstaatsforschung tut sich etwas schwer mit den fordernden Aspekten und den wirtschaftlichen

4 Dies gilt besonders für die Einkommensteuerfreibeträge, von denen vor allem besser verdienende Eltern profitierten (vgl. Schulz 1998, S. 141f.).

5 Die Autonomieverlust-Diagnose bezieht sich zumeist auch nicht auf die materiell-inhaltliche Seite der Sozialpolitik, sondern auf Veränderungen in den Akteurskonstellationen und den politischen Prozessen in den sozialpolitischen Politikfeldern. Sie leidet darunter, dass sie nicht zeigt, wie genau sich diese auf sozialpolitische Inhalte auswirkt.

6 Adalbert Evers etwa argumentiert (2008, S. 242), dass in der Nachkriegszeit "bestimmte wachstums- und integrationsförderliche Dispositionen der Arbeiter, Bürger vorausgesetzt werden" konnten, sodass sozialpolitische Maßnahmengestaltung sich seltener an den gesellschaftlichen Realitäten rieb und daher weniger als "Fordern" in Erscheinung trat. Das sollte jedoch nicht dazu führen, den Forderungscharakter der Sozialpolitik insgesamt infrage zu stellen. 
und politischen Nebenzielen in der Sozialpolitik. Wohlfahrtsstaaten werden oft nur von ihrer Leistungsseite her betrachtet, während Gegenleistungen allenfalls relativ abstrakt, als fiskalische Belastung oder Lohnnebenkosten, in Erscheinung treten. Eine bestimmte Rezeption von Thomas $H$. Marshalls (1950) Vorstellung sozialer Rechte hat in diesem Punkt die vergleichende Wohlfahrtsstaatsforschung stark geprägt. Staatlich organisierte Daseinsvorsorge ist in der Marshall-Perspektive der Versuch, soziale Staatsbürgerschaft, d.h. das Recht auf staatsbürgerliche Teilhabe, zu verwirklichen. Einhergehend mit dem Erkenntnisinteresse an subjektiven Rechtsansprüchen kennzeichnet die Marshall-Tradition eine Fortschrittshoffnung, die in der (Höher-) Entwicklung von Wohlfahrtsstaaten demokratische und emanzipatorische Erfolge erblickte, die in den 1950er bis 1970er Jahren ihren Zenit erreichten und seitdem nur noch Kürzungen und „Retrenchment" erfuhren.

Die Analyse der fordernden Elemente in der Sozialpolitik legt allerdings eine alternative Sicht auf die Sozialpolitik und das Verhältnis zwischen Sozialreformern und „ihren“ Bürgern nahe. In dieser Perspektive sind die Einwohner eines Landes nicht in erster Linie Staatsbürger, und damit Träger sozialer Rechte, sondern es sind „Soldiers and Mothers“ (Skocpol 1992), Steuerzahler, Arbeitskräfte und Erzieher(innen) und damit Produzenten sozialstaatlich relevanter Ressourcen. Das liberale Insistieren auf Rechtsansprüchen gegenüber dem Staat wird durch diese Perspektive ergänzt durch ein, wie Margaret Somers (2005, S. 443ff.) es nennt, „republikanisches“ Interesse an dem aktiven Beitrag, den die Rechtssubjekte in ihrem Gemeinwesen leisten. Sozialpolitisch gewährleistete Teilhabe hat ihre Kehrseite in ebenfalls sozialpolitisch eingeforderter Teilnahme. ${ }^{8}$ Der Gedanke findet seine Entsprechung im aktuellen politischen Diskurs: Viele zeitgenössische alarmistische Kommentare zum demografischen Wandel fragen sich zuerst, wer die Kinder erziehen wird und wer die Sozialversicherung finanzieren soll, und nicht, wer welche Rechtsansprüche an den Staat hat. Stefan Lessenich (2008, S. 17) analysiert das derzeitige „Fordern und Fördern“ in der Sozialpolitik und sieht hierin eine "Neuerfindung des Sozialen“, nach der „sozial“ nicht mehr die kollektive Absicherung individueller Bedürfnisse ist, sondern das eigenverantwortliche, der Allgemeinheit dienliche Verhalten.

\subsection{ZUR ERKLÄRUNG DER FORDERNDEN SOZIALPOLITIK}

Die selektive Lesart von Thomas Marshalls sozialen Staatsbürgerrechten, die einseitig wohlfahrtsstaatliche Leistungen betont, doch nichts mit den mit staatsbürgerlicher Inklusion einhergehenden Pflichten anfangen kann, hat auch den Modus sozialwissenschaftlicher Erklärungen sozialpolitischer Anreize geprägt. Solange die Sozialpolitik vorwiegend in der Perspektive von Rechtsansprüchen gegenüber dem Staat gesehen wird, werden immer zuerst gesellschaftliche Interessen, ihre Mobilisierung und ihre Machtressourcen im Zentrum der Aufmerksamkeit stehen. Oft lassen sich Sozialreformen jedoch nicht eindeutig als Antwort auf die Forderungen gesellschaftlicher Gruppen verstehen - zu diffus und unorganisiert sind die nutznießenden Gruppen, zu unspezifisch ist in manchen Fällen auch der Nutzen selber, $\mathrm{zu}$ einschneidend sind allerdings vielfach die sozialpolitisch kommunizierten Verhaltensanforderungen. All das stellt die Verteilungsdimensionen der Sozialpolitik nicht in Abrede, doch es verweist darauf, dass die Motive hinter sozialreformerischen Aktivitäten sich nicht in Verteilungsinteressen erschöpfen.

Zum einen impliziert dies, dass politische Eliten, deren Verhalten und Politikziele sich nicht ausschließlich als Ausdruck eigener Interessen an Sozialtransfers verstehen lassen, zentral für die Erklärung der fordernden Sozialpolitik werden. Bei Spitzenpolitikern in Regierung und Ministerien kann man der Frage nachgehen, ob ein organisatorisches Eigeninteresse von Akteuren - oder, wie Claus Offe (2006 [1975], S. 130) es formuliert hat, ein „Interesse des Staates an sich selbst" - die Handlungen dieser Personen prägt. Theoretisch ist dieses „Interesse des Staates an sich selbst“ etwas schwer zu fassen, und es ist dann zuweilen schwierig, diese abstrakte Kategorie auf konkrete Inhalte von Sozialreformen zu beziehen. Auf der anderen Seite sind sozialpolitische Diskussionen voll von Formulierungen über angemessenes und produktives Verhalten - zumeist anderer Leute -, Auffassungen über förderungswürdige Lebensweisen und Anschauungen über die richtigen sozialpolitischen Anreize. All das beeinflusst Sozialreformen sicherlich und ganz konkret, doch die Herkunft dieser Positionen lässt sich mitunter nicht sinnvoll auf gesellschaftliche Interessenlagen zurückführen. Die Analyse der fordernden Sozialpolitik weist damit in die Richtung einer staatszentrierten Betrachtungsweise. ${ }^{9}$

Eine zweite theoretische Baustelle im Verständnis der fordernden Sozialpolitik weist in die entgegengesetzte Richtung. Organisierte gesellschaftliche Gruppen spielen für das Verständnis der fordernden Elemente in der Sozialpolitik vielleicht nicht die zentrale Rolle, die sie bei den umverteilenden Elementen spielen. Doch Staatsbürger können die Politik durch unkoordinierte individuelle Verhaltensweisen und Entscheidungen - bspw. Entscheidungen gegen Kinder bei einer relevanten Minderheit von Frauen, Bildungsmüdigkeit bei einer relevanten Minderheit von Schülern, etc. - unter Druck setzen. Dieses „Drohpotenzial“ braucht keine politische Mobilisierung, doch die hektischen Versuche, beispielsweise die Vereinbarkeit zwischen Familie und Beruf zu verbessern oder Bildung und Integration fördernd und fordernd für alle Einwohner des Landes zur Norm und Realität zu machen, sind ein Hinweis auf deren Wirkungsmächtigkeit. In der politischen Ökonomie ist diese Art von „struktureller Macht“ altbekannt (Lindblom 1982), doch sie wird landläufig nur den Arbeitgebern zugeschrieben: Diese können Investitionen verweigern, damit großen Schaden anrichten und die Politik unter Druck setzen. Bürgern und Arbeitnehmern hat man zumeist das Drohpotenzial unkoordinierten, ohne politische Mobilisierung auskommenden Einflusses auf die Politik abgesprochen ${ }^{10}$ und darin gerade die Machtasymmetrie zwischen Kapital und Arbeit begründet gesehen (Offe 1981, S. 146ff.).

Vor allem die familienpolitischen Reaktionen auf den Geburtenrückgang verdeutlichen aber nun, dass auch das unkoordinierte Massenverhalten von Frauen, Arbeitnehmern, Auszubildenden und Bürgern in einigen Bereichen die Politik erheblich unter Druck setzen kann. Leonhard Schoppa entwickelt ein Modell

7 George Steinmetz (1993, S. 31) hat diese Vorstellung treffend als "Whig narrative of social progress" bezeichnet.

8 Den Doppelcharakter von sozialer Inklusion als Teilhabe und Teilnahme betont auch Martin Kronauer (2002).

9 Diesen Punkt habe ich an anderer Stelle eingehender behandelt (Mätzke 2011).

10 Der einzige machtvolle Akt der unorganisierten Bevölkerung besteht im Gang zur Wahlurne, doch davor haben die Parteien - Organisationen - bereits über die politische Agenda entschieden. 
der unkoordinierten Entscheidungen über Elternschaft und Erwerbsbeteiligung, um mit dessen Hilfe familienpolitische Initiativen (oder ihr Fehlen) zu erklären (Schoppa 2005). Doch es wird dort auch deutlich, dass sich dieses Verhalten nicht in gleicher Weise wie der „Investitionsstreik“ der Arbeitgeber in strukturelle Macht übersetzt, weil es Politikern schwer fällt, die diffusen Signale unkoordinierten Massenverhaltens richtig zu deuten, und weil sich das Drohpotenzial des „Geburtenstreiks“ über Zeiträume aufbaut, nach denen es dann für politische Reaktionen bereits zu spät ist. Dennoch ist es für das Verständnis des Verhältnisses zwischen Staat und Bürgern wichtig, die Möglichkeit und die Wirkungsmächtigkeit individueller Entscheidungen im Auge zu behalten. Gerade hier wollen ja viele der sozialpolitischen Anreizpolitiken ansetzen.

Der Blick auf die fordernden Elemente in der Sozialpolitik lenkt unsere Aufmerksamkeit also auf zwei Wirkungsmechanismen, die in der Erklärung sozialpolitischer Entwicklungen bislang noch etwas im Schatten der vielen Theorien über organisierte gesellschaftliche Interessen stehen: Sozialreformerische Entscheidungen politischer Entscheidungsträger, die nicht unmittelbar auf gesellschaftliche Interessenlagen zurückzuführen sind und das Einflusspotenzial individuellen, unkoordinierten Massenverhaltens. Die Anreizelemente in sozialpolitischen Maßnahmen bieten eine Gelegenheit, diese wichtigen Bestandteile von Erklärungen der Sozialpolitik genauer zu betrachten. Darin liegt ihre Bedeutung jenseits der bloßen Beobachtung, dass der Staat nicht nur verteilt, sondern auch fördert und fordert.

\section{LITERATUR}

Baldwin, P. (2005): Beyond Weak and Strong: Rethinking the State in Comparative Policy History, in: The Journal of Policy History 17 (1),

S. $12-33$

Bertram, H./Krüger, H./Spieß, C. K. (Hrsg.) (2006): Wem gehört die Familie der Zukunft? Expertisen zum 7. Familienbericht der Bundesregierung, Opladen

Braun, H. (1978): Das Streben nach "Sicherheit“ in den 50er Jahren.

Soziale und Politische Ursachen und Erscheinungsweisen, in: Archiv für Sozialgeschichte 18, S. 279-306

Esping-Andersen, G. (1990): The Three Worlds of Welfare Capitalism, Princeton, New Jersey

Estévez-Abe, M./Iversen, T./Soskice, D. (2001): Social Protection and the Formation of Skills: A Reinterpretation of the Welfare State, in: Hall, P. A./Soskice, D. (Hrsg.): Varieties of Capitalism. The Institutional Foundations of Comparative Advantage, Oxford/New York, S. 145-183 Evers, A. (2008): Investiv und aktivierend oder ökonomistisch und bevormundend? Zur Auseinandersetzung mit einer neuen Generation von Sozialpolitiken, in: Evers, A./Heinze, R. G. (Hrsg.): Sozialpolitik: Ökonomisierung und Entgrenzung, Wiesbaden, S. 229-250

Evers, A./Heinze, R. G. (Hrsg.) (2008): Sozialpolitik: Ökonomisierung und Entgrenzung, Wiesbaden

Henninger, A./Wimbauer, C./Dombrowski, R. (2008): Geschlechtergleichheit oder „exklusive Emanzipation“? Ungleichheitssoziologische Implikationen der aktuellen familienpolitischen Reformen, in: Berliner Journal für Soziologie 18 (1), S. 99-128

Hinrichs, K. (2004): Alterssicherungspolitik in Deutschland: Zwischen Kontinuität und Paradigmenwechsel, in: Stykow, P./Beyer, J. (Hrsg.):

Gesellschaft mit beschränkter Hoffnung: Reformfähigkeit und die Möglichkeit rationaler Politik, Wiesbaden, S. 266-286

Hockerts, H. G. (1980): Sozialpolitische Entscheidungen im Nachkriegsdeutschland. Alliierte und deutsche Sozialversicherungspolitik 1945 bis 1957, Stuttgart

Hockerts, H. G. (1986): Integration der Gesellschaft: Gründungskrise und Sozialpolitik in der frühen Bundesrepublik, in: Zeitschrift für Sozialreform 32 (1), S. 25-41
Höpner, M. (2006): Determinanten der Quersubventionierung. Ein Vorschlag zur Analyse wirtschaftlicher Liberalisierung, in: Berliner Journal für Soziologie 16 (1), S. 7-23

Jantz, K. (1977): Die Rentendynamik 1957 als Vorbild im Sozialleistungsrecht, in: Bartholomäi, R./Bodenbender, W./Henkel, H./Hüttel, R. (Hrsg.): Sozialpolitik nach 1945. Geschichte und Analysen, Bonn-Bad Godesberg, S. 109-123

Kemmerling, A./Bruttel, O. (2006): "New” Politics in German Labour Market Policy? The Implications of the Recent Hartz Reforms for the German Welfare State, in: West European Politics 29 (1), S. 90-112 Kronauer, M. (2002): Exklusion. Die Gefährdung des Sozialen im hoch entwickelten Kapitalismus, Frankfurt/M.

Lessenich, S. (2008): Die Neuerfindung des Sozialen. Der Sozialstaat im flexiblen Kapitalismus, Bielefeld

Lindblom, C. E. (1982): The Market as Prison, in: The Journal of Politics 44 (2), S. 324-336

Manow, P. (2001): Social Protection, Capitalist Production. The Bismarckian Welfare State and the German Political Economy from the 1880 s to the 1990s, University of Konstanz

Marshall, T. H. (1950): Citizenship and Social Class, London

Mätzke, M. (2011): Staatsbürger als Wirtschaftssubjekte und als demografische Ressource. Die Ziele staatlicher Akteure in der Sozialpolitik, in: Leviathan (Veröffentlichung geplant)

Moeller, R. G. (1993): Protecting Motherhood. Women and the Family in the Politics of Postwar Germany, Berkeley/Los Angeles/Oxford

Mückenberger, U. (1985): Die Krise des Normalarbeitsverhältnisses, in: Zeitschrift für Sozialreform 31 (7), S. 415-434

Offe, C. (1981): The attribution of public status to interest groups: observations on the West German case, in: Berger, S. (Hrsg.): Organizing Interests in Western Europe. Pluralism, Corporatism, and the Transformation of Politics, S. 123-158

Offe, C. (2006 [1975]): Reformpolitik und das Interesse des Staates an sich selbst, in: Borchert, J./Lessenich, S. (Hrsg.): Strukturprobleme des kapitalistischen Staates. Aufsätze zur Politischen Soziologie, Veränderte Neuausgabe, Frankfurt/M., S. 127-152 
Ostner, I. (2006): Paradigmenwechsel in der (west-)deutschen Familienpolitik, in: Berger, P. A./Kahlert, H. (Hrsg.): Der demografische Wandel. Chancen für die Neuorientierung der Geschlechterverhältnisse, Frankfurt/New York, S. 165-199

Ostner, I. (2008): Ökonomisierung der Lebenswelt durch aktivierende Familienpolitik?, in: Evers, A./Heinze, R. G. (Hrsg.): Sozialpolitik. Ökonomisierung und Entgrenzung, Wiesbaden, S. 49-66

Piven, F. F./Cloward, R. A. (1971): Regulating the Poor: The Functions of Public Welfare, New York

Ristau, M. (2005): Der ökonomische Charme der Familie, in: Aus Politik und Zeitgeschichte 23-24 (6. Juni), S. 16-23

Rürup, B./Gruescu, S. (2003): Nachhaltige Familienpolitik im Interesse einer aktiven Bevölkerungspolitik. Gutachten im Auftrag des Bundesministeriums für Familie, Senioren, Frauen und Jugend, Berlin Sachße, C./Tennstedt, F. (1986): Sicherheit und Disziplin: Eine Skizze zur Einführung, in: Sachße, C./Tennstedt, F. (Hrsg.): Soziale Sicherheit und soziale Disziplinierung, Beiträge zu einer historischen Theorie der Sozialpolitik, Frankfurt/M., S. 11-44

Schoppa, L. (2005): Exit, Voice, and Women's Movements in an Era of Low Fertility, Annual Meeting of the American Political Science Association, Washington, DC

Schulz, G. (1998): Soziale Sicherung von Frauen und Familien, in: Hockerts, H. G. (Hrsg.): Drei Wege Deutscher Sozialstaatlichkeit: NSDiktatur, Bundesrepublik und DDR im Vergleich, München, S. 117-149
Skocpol, T. (1992): Protecting Soldiers and Mothers. The Political Origins of Social Policy in the United States, Cambridge/London Somers, M. (2005): Citizenship Troubles. Genealogies of Struggle for the Soul of the Social, in: Adams, J./Clemens, E.S./Orloff, A.S. (Hrsg.): Remaking Modernity. Politics, History, and Sociology, Durham/London, S. $438-469$

Steinmetz, G. (1993): Regulating the Social. The Welfare State and Local Politics in Imperial Germany, Princeton

Streeck, W. (2001): High Equality, Low Activity: The Contribution of the Social Welfare System to the Stability of the Collective Bargaining Regime, EUI Working Paper 6

Trampusch, C. (2006): Status quo vadis?: Die Pluralisierung und Liberalisierung der "Social-Politik": eine Herausforderung für die politikwissenschaftliche und soziologische Sozialpolitikforschung, in: Zeitschrift für Sozialreform 52 (3), S. 299-323

Wuermeling, F. J. (1956): Um den Familienlastenausgleich, in: Die Neue Ordnung in Kirche, Staat, Gesellschaft, Kultur 10 (5), S. 257-267

Wuermeling, F. J. (1963): Der besondere Schutz der Familie durch die staatliche Ordnung, in: Wuermeling, F. J. (Hrsg.): Familie - Gabe und Aufgabe, Köln 